\title{
EVIDÊNCIA DA ATUAÇÃO DO SISTEMA DE AUTO-INCOMPATIBILIDADE TARDIA EM ACCA SELLOWIANA (BERG) BURRET. (MYRTACEAE) ${ }^{1}$
}

\author{
KARINE LOUISE DOS SANTOS ${ }^{2}$, MAURÍCIO LENZI ${ }^{3}$, CLARISSA ALVES CAPRESTANO $^{4}$, \\ ADRIANA CIBELE DE MESQUITA DANTAS ${ }^{5}$, JEAN PIERRE HENRI JOSEPH DUCROQUET ${ }^{6}$, \\ RUBENS ONOFRE NODARI ${ }^{5}$, AFONSO INÁCIO ORTH ${ }^{5}$, MIGUEL PEDRO GUERRA $^{5}$.
}

\begin{abstract}
RESUMO-Acca sellowiana (Myrtaceae) é uma frutífera nativa da região Sul do Brasil e nordeste do Uruguai, que vem despertando grande interesse devido ao alto potencial organoléptico de seus frutos. Neste trabalho, teve-se como objetivo a caracterização do tipo de sistema de incompatibilidade atuante em A. sellowiana, através da avaliação do desenvolvimento dos tubos polínicos. Utilizaramse dois acessos: 458, sendo autocompatível, e 101, auto-incompatível. A maior porcentagem de germinação de grãos de pólen foi observada no acesso 101, com 68,8\% de grãos de pólen germinados. Não foram observadas diferenças no crescimento dos tubos polínicos em pistilos autopolinizados ou de polinização cruzada, em ambos os acessos. O crescimento completo do tubo polínico até o ovário ocorreu em pistilos coletados 96 horas após a polinização, independentemente do tratamento aplicado. Sugere-se a ocorrência de auto-esterilidade ou auto-incompatibilidade tardia ou pós-zigótica, considerando que o abortamento dos frutos de A. sellowiana é uniforme e ocorre num prazo curto, por volta de 20 a 30 dias após a fertilização das flores.
\end{abstract}

Termos para indexação: sistema reprodutivo, auto-incompatibilidade, polinização, fluorescência.

\section{EVIDENCE OF THE LATE-ACTING SELF-INCOMPATIBILITY SYSTEM IN ACCA SELLOWIANA (BERG) BURRET. (MYRTACEAE)}

\begin{abstract}
Acca sellowiana (Myrtaceae) is a native fruit species from the South Brazil and northeast Uruguay which is attracting great interest due to the high organoleptic potential of its fruits. In this study we aimed to characterize the type of incompatibility system which occurs in A. sellowiana, through the evaluation of the polinic tube development. Two accesses were used: being 458 self-compatible and 101 self- incompatible. The highest percentage of pollen grains germination, $68,8 \%$, was observed in the access 101. It was not observed differences in the growth of the polinic tubes between self-pollinated pistils and cross-pollinated ones in both accesses. The complete growth of the polinic tube until reaching the ovary occurred only in pistils collected 96 hours after the polinization, independent of the treatment. The data suggests the occurrence of self-sterility, or a delayed or post-zygotic selfincompatibility in the species, considering that the abortion of A. sellowiana fruits is uniform and occurs in a short period of time, around 20 to 30 days, after the fertilization of the flowers.
\end{abstract}

Index terms: breeding systems, self-incompatibility, pollination, fluorescence.

\section{INTRODUÇÃO}

Acca sellowiana (Berg) Burret. (Myrtaceae), também conhecida regionalmente como goiabeira-serrana, é uma fruteira nativa da região Sul do Brasil e nordeste do Uruguai, que vem despertando grande interesse econômico devido ao alto potencial organoléptico de seus frutos. Por meio de ações multidisciplinares, baseadas nas áreas de biologia celular e molecular, biologia reprodutiva e conservação, tem-se objetivado intensificar o processo de domesticação desta espécie. Entretanto, estudos sobre a biologia reprodutiva da goiabeiraserrana são escassos; todavia, mostram-se prioritários para o processo do programa de melhoramento genético da espécie.

As flores da A. Sellowiana são bispóricas e longistiladas, com tendência a dicogamia por protoginia, uma vez que o estigma se encontra receptivo 24 horas antes da deiscência das anteras e por mais dez horas após a deiscência das anteras (Mattos,1986;
Stewart \& Craig, 1989; Degenhardt et al., 2001), indicando a alogamia como sistema de polinização preferencial.

$\mathrm{O}$ entendimento do sistema de auto-incompatibilidade pode gerar informações úteis no planejamento de estratégias de melhoramento, hibridações controladas e composição de pomares, os quais venham a garantir uma adequada polinização com o uso de genótipos compatíveis. Neste contexto, o objetivo deste estudo foi caracterizar a presença de mecanismos de autoincompatibilidade atuante em A. sellowiana, com o auxílio da técnica de epifluorescência associada a estudos de germinação e viabilidade do pólen e frutificação efetiva.

\section{MATERIAL E MÉTODOS}

Foram utilizados dois acessos distintos pertencentes a diferentes classes de compatibilidade: 458-autocompatível e 101auto-incompatível, mantidos em triplicata no Banco Ativo de

\footnotetext{
${ }^{1}$ (Trabalho 136-2006). Recebido em 12-09-2006. Aceito para publicação em 13-2-2007.

2 Eng. Agrônoma, M.Sc., Centro de Ciências Agrárias, Depto. Fitotecnia, Programa de Pós-Graduação em Recursos Genéticos Vegetais, UFSC, CEP 88034-001, Florianópolis-SC. Fone. (0xx48) 331.5333. e-mail: karinesantos@cca.ufsc.br

Biólogo, M.Sc., Programa de Pós-Graduação em Recursos Genéticos Vegetais, CCA/UFSC. e-mail: mlenzi_pgrgv@yahoo.com.br

${ }^{4}$ Eng. Agrônoma, Programa de Pós-Graduação em Recursos Genéticos Vegetais, CCA/UFSC. e-mail: Clarissa@duol.com.br

${ }^{5}$ Eng. Agrônomo, Drs., Centro de Ciências Agrárias, Depto. Fitotecnia, Programa de Pós-Graduação em Recursos Genéticos Vegetais, UFSC.

${ }^{6}$ Pesquisador Empresa de Pesquisa Agropecuária e Extensão rural de Santa Catarina S.A (EPAGRI)
} 
Germoplasma de São Joaquim (SC). Para a germinação dos grãos de pólen, foi seguida a metodologia proposta por Franzon et al. (2005), onde, na pré-antese, flores previamente protegidas foram coletadas, e suas anteras, removidas e mantidas em estufa BOD a $24^{\circ} \mathrm{C}$ por 24 horas até a completa deiscência, sendo os grãos de pólen mantidos a $4^{\circ} \mathrm{C}$. O acompanhamento da germinação dos grãos de pólen foi efetuado quatro e seis horas após o início do experimento, sob microscópio óptico de luz (100x). O delineamento experimental utilizado para acompanhamento da germinação do pólen foi inteiramente casualizado, com quatro repetições, tendo como tratamentos o tempo de incubação em meio de cultura (quatro e seis horas). Os dados foram submetidos ao teste T, a $5 \%$ de significância.

Para a avaliação do desenvolvimento do tubo polínico no pistilo das flores, foi realizada a polinização manual das flores, em quatro tratamentos: $101 \times 101 ; 101 \times 458 ; 458 \times 458$ e $458 \times 101$. Em cada tratamento, 50 flores de cada acesso foram previamente ensacadas e emasculadas. Foram, também, coletados 10 pistilos de cada tratamento em intervalos regulares de 12; 24; 48 e 96 horas após a polinização, fixados em FAA 50\%, e posteriormente corados e avaliados, seguindo-se a metodologia proposta por Martin (1959). As observações foram feitas em microscópio invertido Olympus IMT-2 com aparato de epifluorescência, com filtros de $450 \mathrm{~nm}$.

Foram realizadas avaliações da frutificação efetiva dos cruzamentos realizados em 40 flores polinizadas, para cada um dos quatro tratamentos citados anteriormente, realizando-se a avaliação da porcentagem de frutificação efetiva aos 50 dias após a polinização.

\section{RESULTADOS E DISCUSSÃO}

Os resultados obtidos nas contagens de grãos de pólen germinados demonstraram maior porcentagem de germinação no acesso 101 , com média de $68,8 \%$ de grãos de pólen germinados. No acesso 458, a média foi de $42 \%$ (Fig. 1). Embora o porcentual de germinação do acesso 458 tenha sido inferior ao obtido no acesso 101 e a média $(59,9 \%)$ obtida por Franzon et al. (2005), não foi observada diferença qualitativa significativa na abundância de tubos polínicos desenvolvidos em pistilos de ambos os acessos. Neste sentido, ressalta-se que a viabilidade apresentada pelos grãos de pólen de ambos os acessos avaliados, segundo os parâmetros acima citados, foi suficiente para realização de polinizações bem-sucedidas.

Ambos os acessos avaliados apresentaram acréscimo na porcentagem de grãos de pólen germinados após seis horas de incubação. No entanto, não houve diferença significativa na porcentagem de germinação, tendo por variável o tempo de incubação (Tab. 1). Do mesmo modo, não houve interação significativa entre acessos e tempos de incubação.

O crescimento dos tubos polínicos foi semelhante tanto em pistilos autopolinizados como naqueles de polinização cruzada, em ambos os acessos e nos diferentes horários de coleta após a polinização. Em geral, o crescimento do tubo polínico, após 12 horas, limitava-se à superfície estigmática ou ao início do primeiro terço do pistilo (Fig. 2 A). Com 24 horas, e em todos os tratamentos, o tubo ainda percorria o primeiro terço do pistilo
(Fig. 2 B) e, com 48 horas, encontrava-se, em média, na região central do pistilo (Fig. 2 C). O crescimento completo do tubo polínico até o ovário foi observado apenas em pistilos coletados 96 horas após a polinização. Não foi possível observar claramente a entrada do tubo polínico na micrópila do óvulo (Fig. 2 D). Em algumas espécies pertencentes às famílias Apocynaceae (Lipow \& Wyatt, 1999), Bignoniaceae (Gibbs \& Bianchi, 1999) e Myrtaceae (Pound et al., 2003), igualmente não é observada diferença quanto ao crescimento do tubo polínico entre plantas compatíveis e incompatíveis.

Entre os tratamentos $458 \times 458$ e $458 \times 101$, não houve diferença na porcentagem de frutificação efetiva ( $50 \%$ em ambos). No tratamento $101 \times 458$, a porcentagem de frutificação foi de $72,5 \%$ enquanto para o tratamento $101 \times 101$, por tratar-se de um acesso auto-incompatível, não foi verificada frutificação.

Considerando-se os resultados obtidos neste experimento, principalmente após ter verificado a presença de tubos polínicos no ovário, pode-se sugerir que não existe um sistema clássico de auto-incompatibilidade que impeça a germinação dos grãos de pólen ou crescimento do tubo polínico para os acessos estudados, tais como a incompatibilidade homomórfica esporofítica, homomórfica gametofítica e heteromórfica, os quais caracterizariam um sistema de incompatibilidade pré-zigótica (Gibbs \& Bianchi, 1999).

Já são bem conhecidos os sistemas de autoincompatibilidade homomórfica e heteromórfica, tanto fisiológica quanto geneticamente. No sistema de incompatibilidade homomórfica gametofítica, a reação de incompatibilidade é determinada pelo alelo $\mathrm{S}$ do gametófito masculino. A herança é geralmente monofatorial e com número variável de alelos. $\mathrm{O}$ reconhecimento do pólen incompatível baseia-se na interação proteína-proteína dos dois determinantes (masculino e feminino), sendo que a resposta de auto-incompatibilidade ocorre quando ambos os determinantes estão carregados com o mesmo haplótipo-S (Takayama \& Isogai, 2005). Na incompatibilidade homomórfica esporofítica, a especificidade do pólen é determinada pelo genótipo diplóide do esporófito que o produziu. O controle genético da incompatibilidade é exercido apenas pelo lócus $S$ com alelos múltiplos (Richards, 1986). Por sua vez, a incompatibilidade heteromórfica está freqüentemente associada com a distilia e tristilia. O termo heteromórfico faz referência à hererostilia, a qual também se caracteriza por as flores diferenciarem-se quanto ao comprimento dos estames (Richards, 1986).

TABELA 1 - Porcentagem média (\%) de grãos de pólen germinados in vitro, em períodos de quatro e seis horas, de dois acessos (101 e 458) de $A$. sellowiana, mantidos na Estação Experimental de São Joaquim, Epagri-SC.

\begin{tabular}{ccc} 
Avaliações & Acesso 101 & Acesso 458 \\
\hline $1^{\circ}$ avaliação -4 horas & $65.05 \mathrm{a}$ & $35.5 \mathrm{~b}$ \\
$2^{\circ}$ avaliação -6 horas & $72.55 \mathrm{a}$ & $48.5 \mathrm{~b}$
\end{tabular}

*Médias na mesma linha seguidas pela mesma letra não são estatisticamente diferentes, pelo teste $\mathrm{T}$, a $5 \%$ de significância 


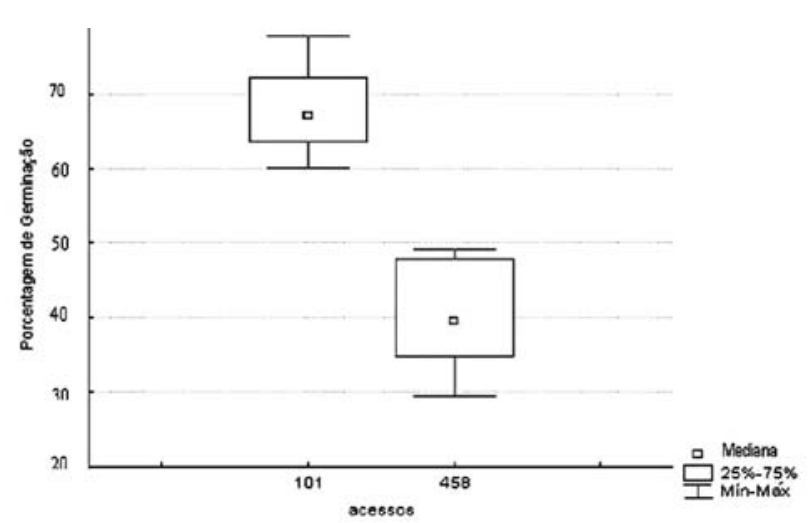

FIGURA 1 - Distribuição das médias e desvio-padrão da porcentagem média de germinação dos grãos de pólen obtidos de $A$. sellowiana, entre dois acessos: 101 e 458 (Teste T, á=0,05\%).

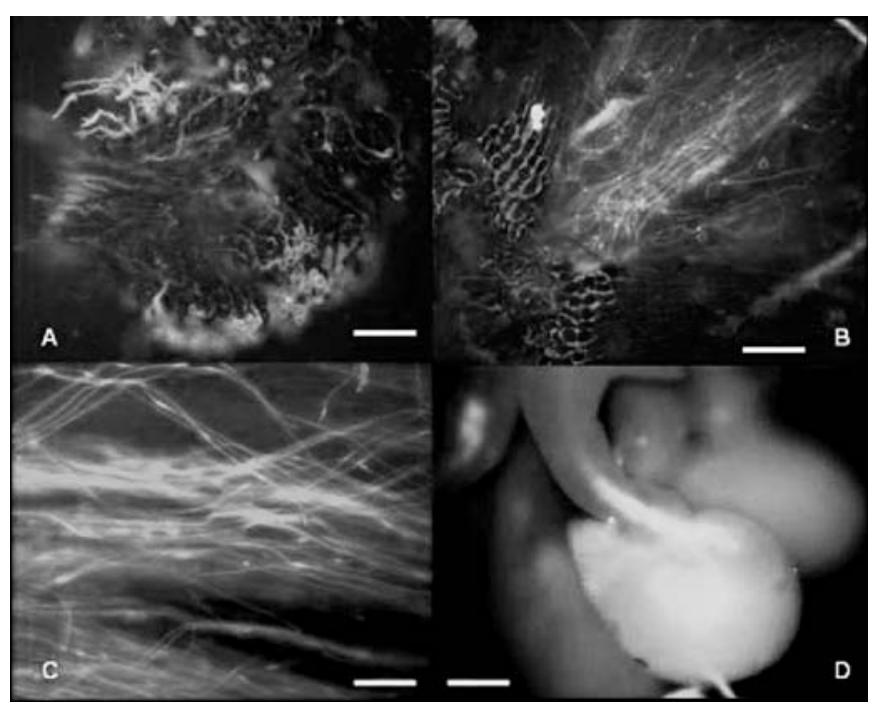

FIGURA 2 - Desenvolvimento dos tubos polínicos de $A$. sellowiana: (A) germinação de grãos de pólen sobre a papila estigmática do pistilo, pertencente ao cruzamento $101 \times 458,12$ horas após a polinização; (B) tubos polínicos no início do primeiro terço do pistilo, pertencente ao cruzamento dos acessos $101 \times 458$, com 24 horas após a polinização; (C) tubos polínicos na região central de pistilo procedentes do cruzamento 101x458, 48 horas após a polinização; (D) óvulo de A. sellowiana com indício de penetração do tubo polínico (área mais clara), procedente do tratamento de autopolinização no acesso 458, com 96 horas após a polinização. Barras de escala de 1 $\mu \mathrm{m}$.

No entanto, existe um sistema diferenciado de autoincompatibilidade no qual frutos provenientes de flores autofertilizadas são abortados, determinando um sistema de incompatibilidade tardia (Seavey \& Bawa, 1986, Machado \& Oliveira, 2000; Kill \& Ranga, 2003).
A auto-incompatibilidade tardia vem sendo observada entre espécies perenes, como Apocynum cannabinum Indian Hemp. (Lipow \& Wyatt, 1999), Casearia grandiflora Camb. (Machado \& Oliveira, 2000), Capparis retusa Griseb. (Bianchi \& Gibbs, 2000), espécies do gênero Kielmeyera (Barros, 2002) e do gênero Eucalyptus (Pound et al,. 2003). Embora amplamente distribuído, pois já foi constatado em 11 ordens, envolvendo 18 famílias, totalizando 59 espécies (Gibbs \& Bianchi, 1999), os mecanismos fisiológico e genético deste tipo de autoincompatibilidade permanecem desconhecidos.

Considerando o fato de que a auto-incompatibilidade tardia pode ser conservada entre taxa (Lipow \& Wyatt, 1999), é razoável propor que este sistema esteja presente em Acca sellowiana.

Existem duas hipóteses para explicar a ocorrência de autoincompatibilidade tardia. Na primeira, o evento poderia ser decorrente de um efeito de depressão endogâmica; já, na segunda hipótese, sugere-se que o efeito de auto-incompatibilidade seja decorrente de resposta pós-zigótica de natureza genética ainda indefinida (Lipow \& Wyatt, 1999; Pound et al, 2003). A observação da uniformidade no abortamento do fruto, quando identificada, pode ser um indicativo de resposta pós-zigótica. Entretanto, se o abortamento dos frutos se dá durante os diferentes estádios de desenvolvimento embrionário, este pode ser um indicativo de depressão endogâmica (Seavey \& Bawa, 1986; Gibbs \& Bianchi, 1999). Em A. sellowiana, tem-se observado que o abortamento dos frutos é uniforme e ocorre num prazo curto após a polinização das flores, por volta de 20 a 30 dias (Pescador, 2004). Desta forma, pode-se propor que o sistema de auto-incompatibilidade atuante na espécie esteja relacionado a uma resposta pós-zigótica. Todavia, ainda não existe consenso com relação à caracterização dos sistemas de auto-incompatibilidade tardia baseados no abortamento de frutos, visto que existem indícios da presença de estímulos resultantes do crescimento do tubo polínico sobre a maturação dos óvulos (Gibbs \& Bianchi, 1999).

É relevante destacar a necessidade de ampliação deste estudo, pois, com o aprimoramento da caracterização do sistema atuante sobre a auto-incompatibilidade, será possível avançar em estudos relativos à ecologia da goiabeira-serrana, bem como aperfeiçoar os métodos de melhoramento e produção.

\section{AGRADECIMENTOS}

À M.Sc. Neusa Steiner, à M.Sc.Gabriela C. C. Inocente e à Maria L. Peixoto.

\section{REFERÊNCIAS}

BARROS, M.A.G. Floração sincrônica e sistemas reprodutivos em quatro espécies de Kielmeyera Mart (Guttiferae). Acta Botanica Brasilica, São Paulo, v. 16, n. 01, p. 113-122, 2002.

BIANCHI, M.B; GIBBS, P.E. Late-acting self-incompatibility in Capparis retusa (Capparaceae), a species of Chaco woodland in NE Argentina. Revista Brasileira de Botânica, São Paulo, v.23, n.4, p. 395-400, 2000. 
DEGENHARDT, J. Variação Fenotípica de Características de plantas e de frutos de Goiabeira-serrana (Acca sellowiana). 2001. (Dissertação) - Faculdade de Agronomia. Universidade Federal de Santa Catarina, Florianópolis, 2001.

FRANZON, R.C.; CORRÊA, E.R.; RASEIRA, M.C.B. In vitro pollen germination of feijoa (Acca sellowiana (Berg) Burret). Crop Breeding and Applied Biotechnology, Londrina, v. 5, n. 2, p. 229-233, 2005.

GIBBS, P.E.; BIANCHI, M.B. Does Late-acting selfincompatibility (LSI) Show family Clustering? Two more species of Bignoniaceae with LSI: Dolichandra cynanchoides and Tabebuia nodosa. Annals of Botany, London, v.84, p.449-457, 1999.

KILL, L.H.P.; RANGA, N.T. Ecologia da polinização de Ipomoea asarifolia (ders.) Roem. \& Schult. (Convolvulaceae) na região semi-árida de Pernambuco. Acta Botanica Brasílica, São Paulo, v.17, n.3, p.355-362, 2003.

LIPOW, S.R.; WYATT, R. Floral morphology and late-acting selfincompatibility in Apocynum cannabinum (Apocynaceae). Plant Systematics Evolution, Vienna, v.219, p.99-109, 1999.

MACHADO, A.O.; OLIVEIRA, P.E.A.M. Biologia floral e reprodutiva de Casearia grandiflora Camb. (Flacourtiaceae). Revista Brasileira de Botânica, São Paulo, v. 23, n. 3, p. 283-290, 2000.

MATTOS, J.R. A Goiabeira-serrana. Porto Alegre: Instituto de Pesquisas de Recursos Naturais Renováveis, 1986. 84p. (Publicação IPRNR, 19)

MARTIN, F. Staining and observaing pollen tubes in the style by means of flurescence. Stain Technology, Baltimore, v.34, p. $125,1959$.

PESCADOR, R. Aspectos fisiológicos-estruturais das embriogêneses zigótica e somática de Feijoa sellowiana Berg (Myrtaceae). 2004. Tese (Doutorado) - Instituto de Biociências, Universidade de São Paulo, São Paulo, 2004.

POUND, L.M.; WALLWORK, M.A.B.; POTTS, B.M.; SEDGLEY, M. Pollen tube growth and early ovule development following self and cross-pollination in Eucalyptus nitens. Sex Plant Reproduction, Zurich, v. 16, p.59-69, 2003.

RICHARDS, A. J. Plant breeding systems. London: George Allen \& Unwin, 1986. 528p.

SEAVEY. S.R.; BAWA, K.S. Late-Acting Self-Incompatibility in Angiospems. Botanical Review, Bronx, v. 52, p-195-219, 1986.

STEWART, A.M.; CRAIG, J.L. Factors affecting pollinator effectiveness in Feijoa sellowiana. New Zealand Journal of Crop and Horticultural Science, Auckland, v. 17, p. 145154, 1989.

TAKAYAMA, S.; ISOGAI A. Self-incompatibility in Plants. Annual Review Plant Biology, Palo Alto, v.56, p.467-489, 2005. 